\title{
Microscopic Adenomyosis
}

\author{
Pietro G Signorile ${ }^{1}$, Rosa Viceconte ${ }^{1}$ and Alfonso Baldi ${ }^{* *}$ \\ ${ }^{1}$ Fondazione Italiana Endometriosi, Rome, Italia \\ 2*Department of Environmental, Biological and Pharmaceutical Sciences and Technologies, Campania University “L. Vanvitelli”, Caserta, Italy
}

${ }^{\star}$ Corresponding author: Alfonso Baldi, Department of Environmental, Biological and Pharmaceutical Sciences and Technologies, Campania University "L. Vanvitelli", Caserta, Italy; Email: alfonsobaldi@tiscali.it

Received: September 03, 2019; Accepted: September 17, 2019; Published: October 06, 2019;

\section{Commentary}

Endometriosis is a frequent, chronic inflammatory estrogendependent gynecological disease characterized by the presence of extrauterine endometrial tissue, that affects up to $10 \%$ of all reproductive-aged women. The incidence increases to $30-50 \%$ in women with chronic pelvic pain and infertility $[1,2]$. Most common sites of the ectopic endometrial-like tissue are the pelvic peritoneum and ovaries, but they can be found also under the peritoneal surface, where endometriosis is strongly associated with pelvic pain symptoms [3]. This disease has a noteworthy morbidity, with harmful effect upon women's social working, personal life, and relations with physicians [4]. Notwithstanding, the pathogenesis, as well as the diagnosis and therapy for endometriosis are still not perfectly delineated [5]. Recently, our group and others have generated convincing experimental data suggesting that perturbation of the fine-tuning of the female genital system development during a critical window of time in fetal life as the pathogenetic event prompting to the progression of endometriosis later in life [6-12].

The lack of knowledge about this disease justifies the fact that, to date, endometriosis is an incredibly under-diagnosed and undertreated disease, with an excessively long-time interval between the commencement of the symptoms and conclusive diagnosis of 8-12 years [1]. This is due to the fact that most of the symptoms are nonspecific and there are no non-invasive diagnostic investigations able to reach a definitive diagnosis [13]. The definite diagnosis of endometriosis can be obtained only by histological examination of the ectopic tissue implants collected by invasive surgical or exploratory procedures [1].

The histologic diagnosis of endometriosis is, usually, quite simple and is based essentially on the recognition of both endometriosic glands and stroma, or at least by one of these two elements [1]. The histological appearance of these elements is straightforward; nevertheless, immunohistochemical staining for cytokeratin markers and for CD10 can aid in identification of glands and stroma in doubtful cases [14]. The different histopathological aspects of endometriosis are well known and have been described in detail in an elegant work of Clement some years ago [14]. Even though the histological diagnosis of endometriosis is relatively easy, also for pathologists who are not experts in this pathology, it has been reported that approximately only $50 \%$ of biopsy specimens from areas suggestive of endometriosis at laparoscopic examination have been proven microscopically to be endometriosis. Since the definitive diagnosis of this disease is based on histological examination, it is important for the correct management of the patients, to avoid false negative results at histology.

This phenomenon is particularly true in the case of adenomyosis, a condition of endometriosis in which the endometrial glands are embedded into the myometrium of the uterus [15]. Based on the Sampson's theory, endometriosis and adenomyosis have been considered for a long time two different clinical entities and it took approximately 80 years to put forward a new theory reunifying their pathogenesis [16]. Indeed, adenomyosis is still considered today an 'elusive' or 'enigmatic' disease because of the struggle in diagnosis, and of the indefinite and vague pattern of symptoms which may accompany it. Nevertheless, the frequent association of adenomyosis with other pelvic pathologies is a further aspect which complicates the understanding of related symptoms [17]. Finally, since the moderate to severe degrees of adenomyosis can be accurately diagnosed preoperatively by good-quality ultrasound or magnetic resonance imaging, it would be desirable in the near future to correlate symptomatology with specific findings on imaging and with pathological data.

In our experience it has happened more than once to review cases, reported as negative for adenomyosis, which showed the presence of microscopic adenomyosis foci that had escaped the observation of the pathologist. As an example, in Figure 1 we show a case of multiple microscopic adenomyosis in the posterior wall of the uterus of patients with endometriosis. Indeed, ultrasound analysis had shown alterations suggestive of adenomyosis of the posterior uterine wall, but the histological analysis of the tissue taken was negative. A careful analysis of the histological preparation, however, showed the presence of microscopic endometriotic glands. Immunohistochemical analysis with cytokeratin antibodies confirmed the epithelial nature of these structures. In Figure 2 we show another case of microscopic adenomyosis, in which two small glandular structures were found in the wall of the uterus, as clearly demonstrated by immunohistochemical analysis for cytokeratin. Interestingly, analysis by CD10 clearly showed that in microscopic adenomyosis the stromal component is absent. 

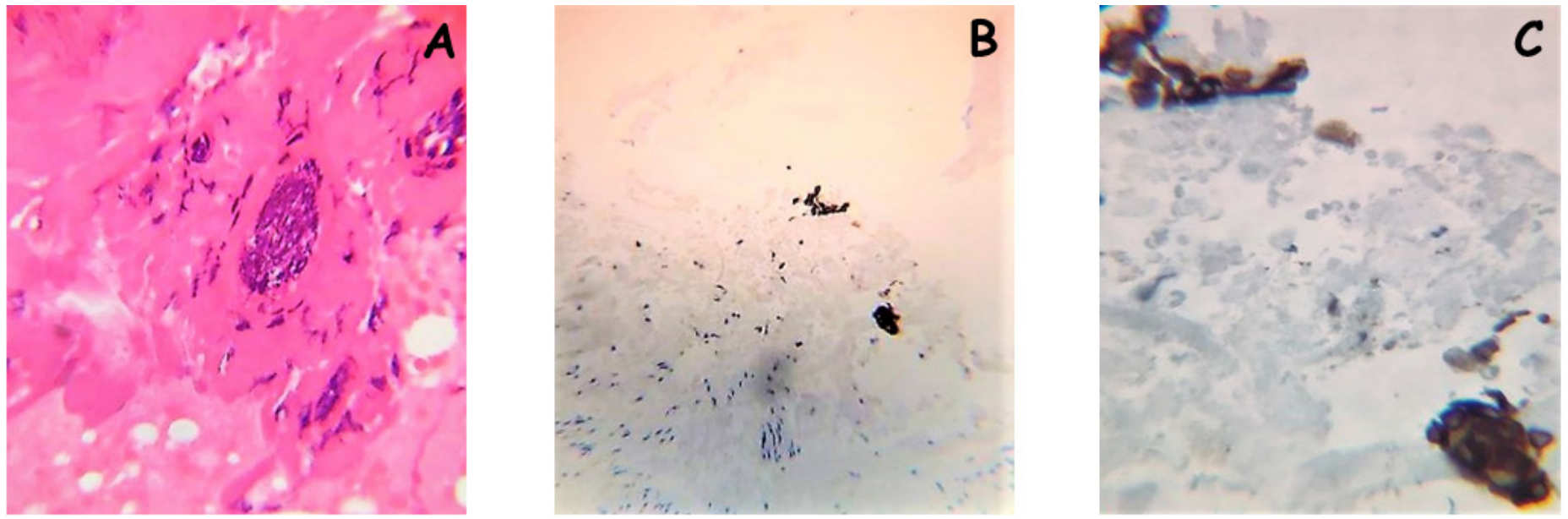

Figure 1. A case of microscopic adenomyosis in the posterior wall of the uterus is depicted. In this case a multifocal microscopic adenomyosis with several very small glands was evidenced A) Histological appearance of the multifocal adenomyosis (Hematoxylin and Eosin; original magnification X20)

B) Immunohistochemical staining for pan-cytokeratin (ABC; original magnification X10)

C) Higher magnification of figure 1B (ABC; original magnification X20)
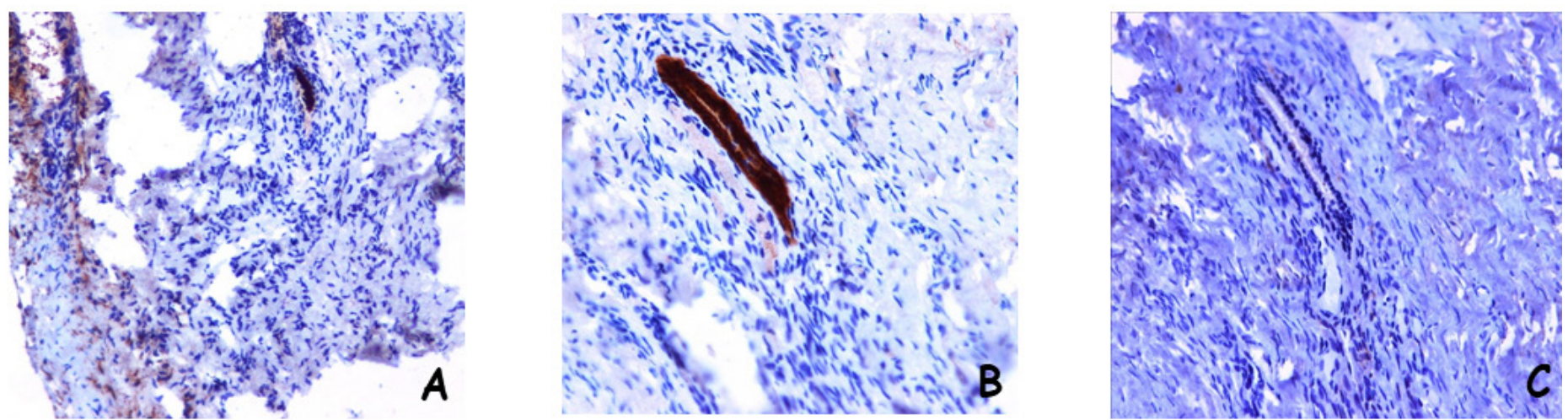

Figure 2. A different case of microscopic adenomyosis in the posterior wall of the uterus is shown. In this case a single small glandular structure was found

A) A small glandular structure evidenced by the immunohistochemical staining for pan-cytokeratin (ABC; original magnification X10)

B) Higher magnification of figure $1^{\circ}(\mathrm{ABC}$; original magnification $\mathrm{X} 20)$

C) The microscopic adenomyosis does not include stroma, as demonstrated by the negative stainining for CD10 (ABC; original magnification X20)

Currently, by means of ultrasound and magnetic resonance imaging analyses, is possible to define for adenomyosis a spectrum of lesions, ranging from increased thickness of the junctional zone to evident adenomyosis and adenomyomas, which in turn can be sub classified [18]. Moreover, it is commonly accepted by the scientific community that adenomyosis is a progressive disease that changes in appearance during the reproductive years. Therefore, it has been recognized the need of a consensus classification of uterine adenomyosis [18].

Based on our experience, microscopic adenomyosis could be considered the earliest form of adenomyosis and should enter the consensus classification of adenomyosis. Furthermore, in the light of this observation, we claim that such an initial state of adenomyosis is a source of symptomatology, thus explaining the presence, as often happens, of patients with negative diagnostic tests but with symptomatology in place, for which even doubts are often raised about the presence of this pathology. Microscopic adenomyosis also provides a rational basis for the occurrence that surgical interventions often do not resolve the symptoms of chronic pelvic pain. Nevertheless, the histological features of microscopic adenomyosis give us clues to the developmental dynamics of endometriosis and adenomyosis. The prevalent glandular-epithelial composition in microscopic adenomyosis may lead to the hypothesis that the role of the stromal component becomes fundamental in a successive phase, providing an essential support to the glandular structures by virtue of its sensitivity to the higher estrogenic growth input with respect to the epithelial component [19]. Finally, we also noted that the greater is tthe multifocal representation of the glands present, the greater is the symptomatic component of pelvic pain.

In conclusion, we propose to consider microscopic adenomyosis as a specific clinical entity and to include it in the classification of uterine adenomyosis Careful histological analysis and, in doubtful cases, the use of immunohistochemistry should always be performed, to eventually confirm the presence of microscopic glands in patients 
with clinical and instrumental signs suggestive for adenomyosis. This would be very important to reduce the delay in the diagnosis of this clinical entity, which is still high today and causes significant problems for both patients and physicians.

\section{References}

1. Bulun SE (2009) Endometriosis. N Engl J Med 360: 268-279.

2. Signorile PG, Campioni M, Vincenzi B, D’Avino A, Baldi A (2009) Rectovaginal septum endometriosis: an immunohistochemical analysis of 62 cases. In Vivo 23: 459-464.

3. Baldi A, Campioni M, Signorile PG(2008) Endometriosis: pathogenesis, diagnosis, therapy and association with cancer. Oncol Rep 19: 843-846.

4. Fuldeore M, Chwalisz K, Marx S, Wu N, Boulanger L, et al. (2001) Surgical procedures and their cost estimates among women with newly diagnosed endometriosis: a US database study. J Med Econ 14: 115-123.

5. Benagiano G, Brosens I (2006) History of adenomyosis. Best Pract Res Clin Obstet Gynaecol 20: 449-463.

6. Signorile PG, Baldi F, Bussani R, D'Armiento M, De Falco M, Baldi A (2009) Ectopic endometrium in human fetuses is a common event and sustains the theory of mullerianosis in the pathogenesis of endometriosis, a disease that predisposes to cancer. J Exp Clin Cancer Res 9: 28-49.

7. Signorile PG, Baldi A (2010) Endometriosis: new concepts in the pathogenesis. Int J Biochem Cell Biol 42: 778-780.

8. Signorile PG, Spugnini EP, Mita L, Mellone P, D’Avino A, et al. (2010) Pre-natal exposure of mice to bisphenol A elicits an endometriosis-like phenotype in female offspring. Gen Comp Endocrinol 168: 318-325.

9. Signorile PG, Baldi F, Bussani R, D’Armiento M, De Falco M, et al. (2010) New evidence of the presence of endometriosis in the human fetus. Reprod Biomed Online 21: 142-147.

10. Signorile PG, Baldi F, Bussani R, Viceconte R, Bulzomi P, et al. (2012) Embryologic origin of endometriosis: analysis of 101 human female fetuses. J Cell Physiol 227: 1653-1656.

11. Bouquet de Jolinière J, Ayoubi JM, Lesec G, Validire P, Goguin A, et al. (2012) Identification of displaced endometrial glands and embryonic duct remnants in female fetal reproductive tract: possible pathogenetic role in endometriotic and pelvic neoplastic processes. Front Physiol 3: 444.

12. Crispi S, Piccolo MT, D’Avino A, Donizetti A, Viceconte R, et al. (2013) Transcriptional profiling of endometriosis tissues identifies genes related to organogenesis defects. J Cell Physiol 228: 1927-1934.

13. Ballard KD, Lowton K, Wright JT (2006) What's the delay? A qualitative study of women's experience of reaching a diagnosis of endometriosis. Fertil Steril 85: 1296-1301.

14. Clement PB (2007) The pathology of endometriosis: a survey of the many faces of a common disease emphasizing diagnostic pitfalls and unusual and newly appreciated aspects. Adv Anat Pathol 14: 241-260.

15. Thylan S Adenomyosis (1995) an ignored uterine disease. Nurse Pract 20: 8-9.

16. Benagiano G, Brosens I, Carrara S. Adenomyosis (2009) new knowledge is generating new treatment strategies. Womens Health (Lond) 5: 297-311.

17. Peric H, Fraser IS (2006) The symptomatology of adenomyosis. Best Pract Res Clin Obstet Gynaecol 20: 547-555.

18. Gordts S, Brosens JJ, Fusi L, Benagiano G, Brosens I (2008) Uterine adenomyosis: a need for uniform terminology and consensus classification. Reprod Biomed Online 17: 244-248.

19. Dyson MT, Kakinuma T, Pavone ME, Monsivais D, Navarro A, et al. (2015) Aberrant expression and localization of deoxyribonucleic acid methyltransferase 3B in endometriotic stromal cells. Fertil Steril 104: 953-963. 